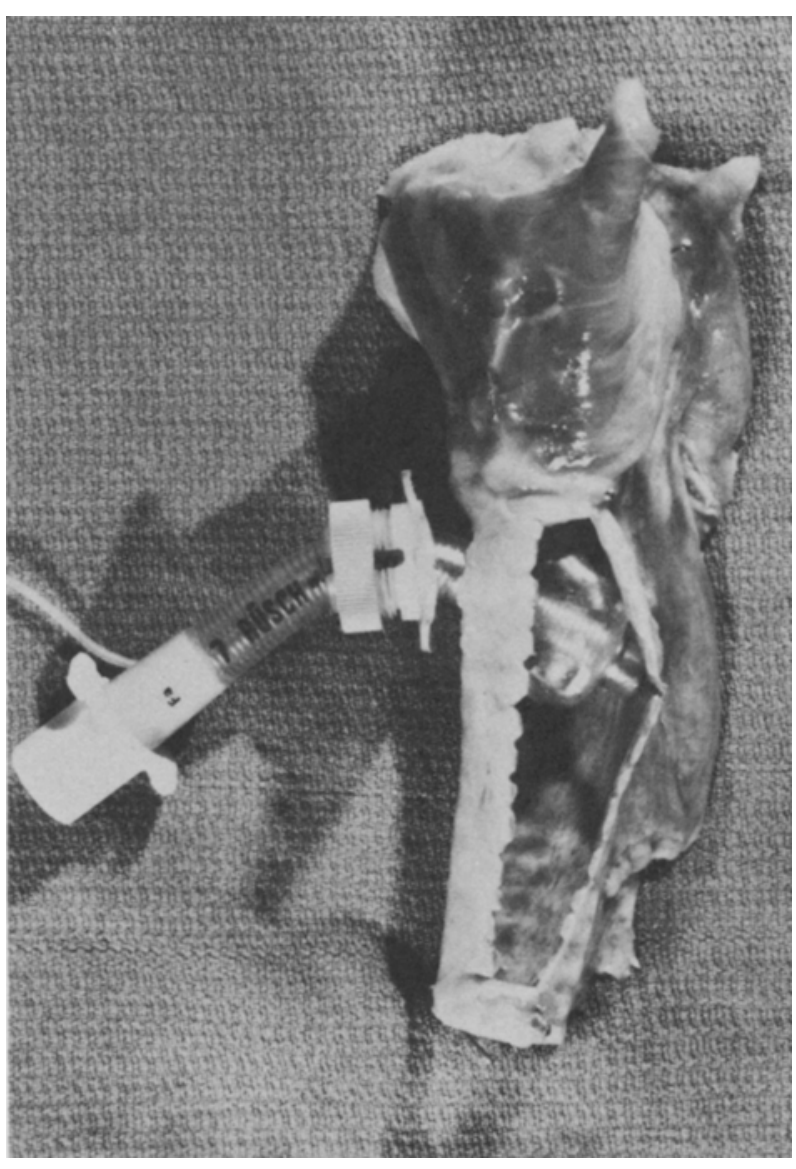

FIGURE 2 Traction on the connector leads to displacement of the cannula within the flange with subsequent tracheal obstruction and inability to ventilate.

accidental traction on the cannula firmly connected to the breathing tubes of the respirator by the built-in connector. We believe that outward displacement of the cannula (1) led to tracheal obstruction, the cannula being perpendicular to the trachea, and (2) rendered ventilation impossible, the distal orifice of the cannula lying against the posterior tracheal wall (Figure 2). Initially it was thought that the locking ring had been insufficiently tightened, but manipulation of the tracheostomy set after the incident disclosed that while maximal tightening of the screw ring made slipping of the cannula more difficult, it could still be moved within the flange quite easily.

While the Rüsch Ultra Tracheoflex ${ }^{\circledR}$ set presents some interesting characteristics such as a built-in connector and a construction designed to minimize tracheal trauma, we believe the design of the restraining collar should be improved. Fenestration of the cannula may help prevent total respiratory obstruction.
We wish to thank Dr. Tack Ki Leung, Department of Pathology, for his collaboration with the illustrations.

\author{
Jean-François Hardy MD FRCPC \\ Jean Taillefer MD FRCPC \\ Yves Hébert MD FRCPC \\ Departments of Anaesthesia and \\ Cardiovascular Surgery \\ Montreal Heart Institute \\ 5000 est, rue Bélanger \\ Montreal, Quebec H1T 1C8
}

\section{REPLY (1)}

Thank you for the opportunity to reply to the letter from Doctors Hardy, Taillefer and Hébert re their experience with the Rusch Ultra Tracheoflex .

Normally it is very difficult to move the adjustable flange on the screw ring once it has been firmly tightened. However, it is possible to do so if the circumference of the cannula is reduced by applying traction to the cannula and stretching it. The softness of the PVC used plus the reinforcing wire in the wall of the tube will permit a small amount of stretching. By applying opposing forces, traction at the machine end (respirator breathing tubes) and pulling on the patient end (the adjustable flange), it is possible to slide the screw ring towards the patient over the reduced circumference of the tube. Continued traction on the collar could allow the cuff to be rotated ... "perpendicular rather than parallel to the axis of the trachea."

With a fixed collar model tracheostomy tube it is probable that these forces would have caused either a disconnect or extubation. It is unfortunate that the very features which benefit the patient, the adjustable flange and soft, flexible cannula, might have been involved in a tragedy. However, it is recognized that the application of abnormal forces to connecting parts can produce abnormal results. That a life-threatening situation was the result in this instance is indeed regrettable and we can all be thankful that the patient experienced an uneventful recovery.

In light of their reported experience we have withdrawn the Ultra Tracheoflex ${ }^{\circledR}$ from the market.

\section{H. Greenberg}

General Manager

Rüsch of Canada Ltd.

\#4 - 2 Select Ave.

Scarborough, Ontario M1V 5J4

\section{REPLY (2)}

The clinical incident reported by Hardy, Taillefer and Hébert on the Rüsch Ultra Tracheoflex ${ }^{\otimes}$ Tracheostomy tube was brought to the attention of the Health Protection Branch, Health and Welfare Canada.

As part of our regulatory assessment, we conducted laboratory simulations of the extubation process to determine the force levels causing the flange to slip on the cannula. An Instron Mechanical Tester pulled on the tracheostomy connector (10 
$\mathrm{cm} \cdot \mathrm{min}^{-1}$ ) while attached to an artificial neck (a plastic beaker $10 \mathrm{~cm}$ in diameter with a $2 \mathrm{~cm}$ diameter tracheostomy). The neck strap was adjusted to a length of approximately $25 \mathrm{~cm}$ which resulted in a moderate tension, similar to that which might be adjusted clinically. The extubation force at which slippage occurred was recorded as the lock nut was adjusted by a varying number of turns. Two new samples (size \#8) and the one involved in the clinical report (size \#7) were tested in this way.

Slippage was observed at a force level of approximately $1 \mathrm{kgf}$ when the lock nut was adjusted by $3 \frac{1}{4}$ to $3 \frac{1}{2}$ turns for the clinical sample and one of the new samples. Although the torque required to tighten the locking nut was not quantified in these simulations, adjusting the locking screw by more than three turns required the flange to be held firmly against the rigid artificial neck. The torque required to tighten the nut became noticeable at about two turns with this new sample, while tightening by more than 3.5 turns required considerable effort. The other new sample required only about $2 \frac{1}{4}$ turns to achieve slippage at these force levels, but the torque was notably higher for the same number of turns.

Most clinical applications are unlikely to exceed the maximum torque levels used in the above simulations (i.e. $3 \frac{1}{2}$ turns), which means that unintentional traction of about $1 \mathrm{kgf}$ can result in the flange slipping with these products. Since breathing circuit disconnects occur at forces greater than $1 \mathrm{kgf}$, clinical incidents involving unintentional traction would be expected to exceed the threshold for slippage. We agree with the clinical report that the design needs to be modified before being considered acceptable for clinical use.

The Canadian distributor promptly issued a recall notice on this product when these concerns were brought to his attention.

\author{
Alex Sinclair PHD \\ Jos Van Bergen \\ Research and Standards Division \\ Bureau of Radiation and Medical Devices \\ Health Protection Branch \\ Health and Welfare Canada \\ Ottawa
}

\section{Determinants of FRC}

To the Editor:

The recent review by Wahba concerning the perioperative functional residual capacity (FRC) ${ }^{1}$ is in keeping with the many excellent educational articles published in this journal. However, I feel a number of statements made by Dr. Wahba require clarification. The first is the statement that partial curarization decreases FRC in supine subjects. Our observations ${ }^{2}$ as well as those of Holle et al. ${ }^{3}$ demonstrated that supine FRC is unchanged by the induction of rather marked degrees of weakness. The study of DeTroyer et al. ${ }^{4}$ cited in the review was performed in seated subjects. In this position the role of tonic inspiratory muscle tone is far more important in maintaining FRC than in the supine position.

The review also cites gas trapping behind closed airways as a major mechanism in the reduction of FRC with general anaesthesia. Rehder et al. ${ }^{5}$ discount this mechanism for FRC reduction since FRC measurements with $\mathrm{N}_{2}$ washout, which measure only gas in contact with open airways, and body plethysmography, which measures the entire volume of thoracic gas including that "trapped," indicated changes of similar magnitude. ${ }^{6}$

The importance of gas trapping is also alluded to in discussion of the effects of tracheal intubation of FRC. Indeed vigorous coughing may be associated with a reduction in FRC following tracheal intubation if gas dilution techniques are utilized. ${ }^{7}$ On the other hand, in patients with moderate airway obstruction, tracheal intubation was accompanied by a significant $(30 \%)$ increase in FRC as measured with helium dilution. ${ }^{8}$ These patients appeared to develop reflex airway constriction but there was no mention of coughing. Tracheal intubation in normal volunteers did not produce significant changes in FRC $^{9,10}$ despite evidence of reflex constriction in airways distal to the tube as suggested by airway resistance measurements. Therefore, there appears to be little reason to speculate that tracheal intubation may reduce FRC either by interfering with glottic action or by causing reflex airway constriction.

Thomas J. Gal MD

Department of Anesthesiology

University of Virginia Health Sciences Center

Charlottesville, VA 22908

\section{REFERENCES}

1 Wahba RWM. Perioperative functional residual capacity. Can J Anaesth 1991; 38: 384-400.

2 Gal TJ, Arora NS. Respiratory mechanics in supine subjects during partial curarization. J Appl Physiol 1982; 53: 57-63.

3 Holle RHO, Schoene RB, Pavlin EG. Effects of respiratory muscle weakness on $\mathrm{P}_{0.1}$ induced by partial curarization. J Appl Physiol 1984; 57: 1150-7.

4 DeTroyer A, Basternier J, Delhez L. Function of respiratory muscles during partial curarization in humans. J Appl Physiol 1980; 47: 1049-56.

5 Rehder K, Cameron PD, Krayer $S$. New dimensions of the respiratory system. Anesthesiology 1985; 62: 230-3.

6 Westbrook PR, Stubbs SE, Sessler AD, Hyatt RE. Effects of anesthesia and muscle paralysis on respiratory mechanics in normal man. J Appl Physiol 1973; 34: 81-6.

7 Bickler PE, Dueck R, Prutow RJ. Effects of barbiturate anesthesia on functional residual capacity and rib cage/diaphragm contributions to ventilation. Anesthesiology $1987 ; 66: 147-52$.

8 Matushima $Y$, Jones $R L$, King EG, Moysa $G$, Alton $J D M$. Alterations in pulmonary mechanics and gas 